\title{
Vaccine for Middle East respiratory syndrome
}

Infection with Middle East respiratory syndrome coronavirus (MERS-CoV) is associated with severe morbidity and mortality, and is not treatable with currently available antiviral therapies. A recent study has shown that a DNA vaccine that targets the spike (S) protein of MERS-CoV is protective against infection in animal models including nonhuman primates and could be useful for the development of preventive strategies in regions affected by the virus.

MERS-CoV is an emerging coronavirus that is distinct from the severe acute respiratory syndrome (SARS)-CoV and shows high levels of genetic diversity.

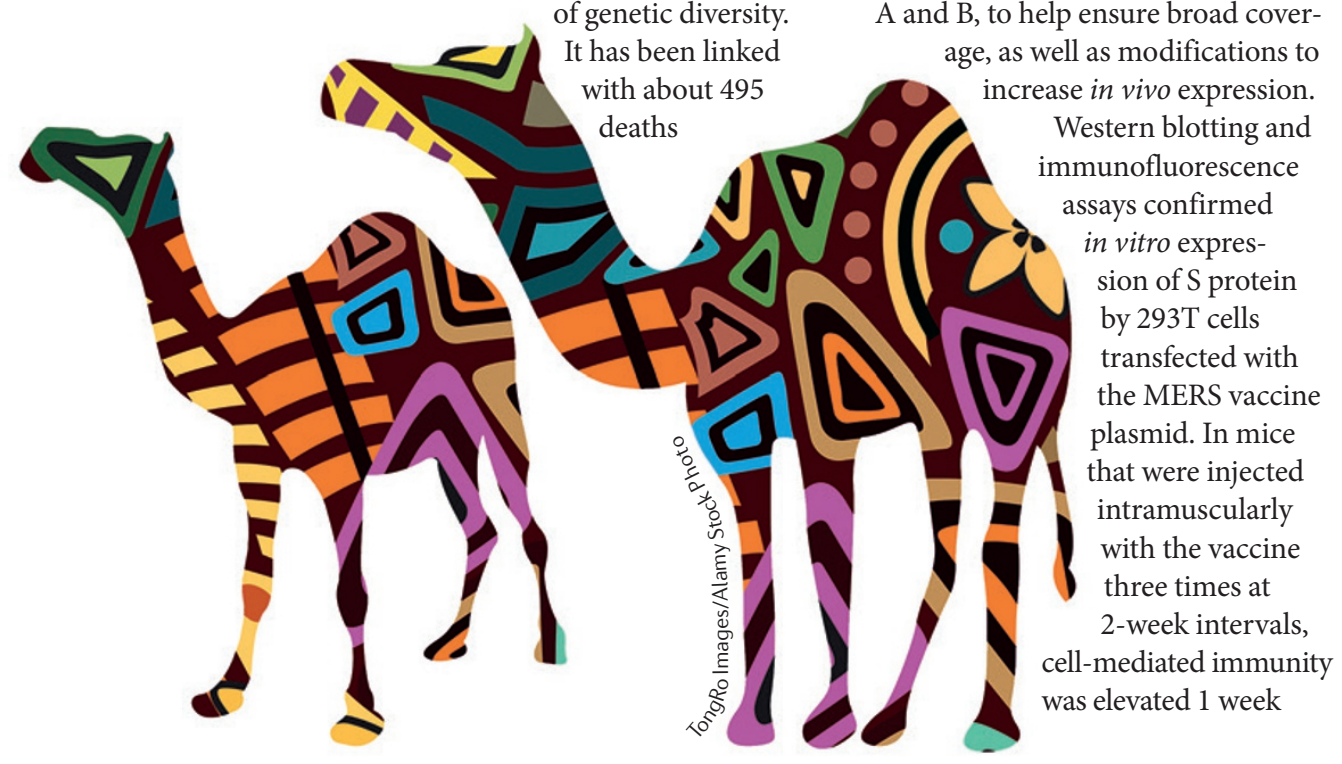

later. $\mathrm{CD} 4^{+}$and $\mathrm{CD} 8^{+} \mathrm{T}$ cells from immunized mice secreted more interferon- $\gamma$, interleukin- 2 and tumour necrosis factor than did cells from vehicle-treated mice, as measured by flow cytometry. Analysis of serum samples before and after immunization revealed that the vaccine induced production of $S$ protein-specific neutralizing antibodies.

Rhesus macaques showed similarly encouraging cellular and humoral responses to vaccination. Importantly, sera from immunized macaques showed neutralizing activity against $\mathrm{S}$ protein from various isolates across both MERS-CoV clades, suggesting that the vaccine could prevent immune escape by genetic variation of the pathogen and thereby provide broad protection. Immunization also protected the macaques from respiratory pathology, such as alveolar oedema and infiltration of inflammatory cells, caused by MERS-CoV challenge 4 weeks after treatment.

Camels are thought to represent a reservoir of MERS-CoV in the Middle East and to be a route of transmission to humans. In the current study, Muthumani et al. show that their DNA vaccine can also induce neutralizing antibodies in camels, suggesting applicability to multipart preventive strategies.

Katie Kingwell

ORIGINAL RESEARCH PAPER

Muthumani, K. et al. A synthetic consensus

anti-spike protein DNA vaccine induces protective immunity against Middle East respiratory syndrome coronavirus in nonhuman primates. Sci. Transl Med. 7, 301ra132 (2015) 